\title{
Comparison of concurrent chemoradiotherapy followed by radical surgery and high-dose-rate intracavitary brachytherapy: a retrospective study of 240 patients with FIGO stage IIB cervical carcinoma
}

This article was published in the following Dove Press journal:

OncoTargets and Therapy

6 January 2014

Number of times this article has been viewed

\author{
Ning Wang' \\ Wei-Wei Li ${ }^{\prime}$ \\ Jian-Ping Li' \\ Juan-Yue Liu' \\ Yong-Chun Zhou' \\ Ying Zhang' \\ Jing $\mathrm{Hu}^{\prime}$ \\ Yan-Hong Huang ${ }^{2}$ \\ Yan Chen ${ }^{3}$ \\ Li-Chun Wei ${ }^{1, *}$ \\ Mei Shil,* \\ 'Department of Radiation Oncology, \\ ${ }^{2}$ Department of Gynecology and \\ Obstetrics, ${ }^{3}$ Department of Oncology, \\ Xijing Hospital, Fourth Military \\ Medical University, Xi'an, People's \\ Republic of China \\ *These authors both contributed \\ equally
}

Background: The aim of this study was to compare the long-term survival outcome and late toxicity in patients with FIGO (International Federation of Gynecology and Obstetrics) stage IIB cervical carcinoma after two treatment modalities, ie, concurrent chemoradiotherapy followed by radical surgery and concurrent chemoradiotherapy followed by high-dose-rate intracavitary brachytherapy.

Methods: Between November 2004 and November 2011, 240 patients with FIGO stage IIB cervical carcinoma were analyzed, comprising 119 patients treated with concurrent chemoradiotherapy followed by radical surgery (group 1) and 121 patients treated with concurrent chemoradiotherapy followed by high-dose-rate intracavitary brachytherapy (group 2). Local control, overall survival, progression-free survival, and treatment-related complications were compared between the two groups.

Results: The median follow-up duration was 36 months. Concurrent chemoradiotherapy followed by radical surgery showed a survival benefit when comparing group 1 and group 2 (3-year overall survival, $94.9 \%$ versus $84.6 \%, P=0.011$; 3-year progression-free survival, $91.0 \%$ versus $81.8 \%, P=0.049$, respectively). Three-year local pelvic control was $94.6 \%$ in group 1 and $93.3 \%$ in group $2(P=0.325)$. Prognostic factors in group 1 were: age ( $\leq 35$ years versus $>35$ years), 3 -year progression-free survival ( $74.1 \%$ versus $90.9 \%, P=0.037$ ); tumor diameter ( $\geq 6 \mathrm{~cm}$ versus $<6 \mathrm{~cm}$ ); and 3-year progression-free survival, (60.6\% versus $92.9 \%, P=0.004)$. Prognostic factors in group 2 were: tumor diameter ( $\geq 4 \mathrm{~cm}$ versus $<4 \mathrm{~cm}$ ); 3 -year overall survival $(78.0 \%$ versus $94.8 \%, P=0.043$ ); tumor diameter ( $\geq 6 \mathrm{~cm}$ versus $<6 \mathrm{~cm}$ ); 3 -year progression-free survival ( $42.9 \%$ versus $84.2 \%, P=0.032$ ); and 3 -year overall survival ( $42.9 \%$ versus $87.1 \%, P=0.013$ ). Further, 50 patients $(42.02 \%)$ in group 1 and 46 patients $(38.02 \%)$ in group 2 suffered from late complications. Analysis of the difference in composition of late complications showed that the rate of leg edema was higher in group $1(35.29 \%$ versus $4.96 \%, P=0.000)$ while the rate of radiation enteritis was higher in group 2 (30.58\% versus $5.04 \%, P=0.000)$.

Conclusion: In patients with FIGO stage IIB cervical carcinoma, concurrent chemoradiotherapy followed by radical surgery achieved higher overall survival and progression-free survival rates in comparison with radical radiotherapy associated with concurrent chemotherapy. Tumor diameter could be a common prognostic factor in these two groups of patients.

Keywords: cervical carcinoma, preoperative concurrent chemoradiotherapy, radical radiotherapy, prognostic factors, late toxicity
Correspondence: Li-Chun Wei; Mei Shi Department of Radiation Oncology, Xijing Hospital, Fourth Military Medical University, 169 Changle West Road, Xi'an 710032, People's Republic of China Tel +862984775432

Email weilichun@fmmu.edu.cn; mshi82@fmmu.edu.cn 


\section{Introduction}

Concurrent cisplatin-based chemoradiotherapy is the standard treatment for locally advanced cervical carcinoma (LACC) based on five clinical trials (GOG85, Radiation Therapy Oncology Group [RTOG] 9001, GOG123, GOG120, SWOG 8797) and two meta-analyses. ${ }^{1,2}$ However, the 5 -year survival of patients with LACC is still around $70 \%{ }^{3}$ In the GOG123 trial, survival status after concurrent chemoradiotherapy followed by radical surgery was better than after radiotherapy alone in patients with FIGO (International Federation of Gynecology and Obstetrics) stage IB2 cervical carcinoma, but for FIGO stage IIB, the large-scale international clinical experimental experience concerning surgical benefit is rare, especially in contrast with radical radiotherapy. Neoadjuvant treatments followed by radical surgery have been proposed, with data for overall survival and disease-free survival reported as ranging from $57 \%$ to $85 \%$ and from $64 \%$ to $90 \%$, respectively. 4,5 More recently, there have been reports that adjuvant surgery after chemoradiotherapy may represent a more effective strategy by removing radiochemoresistant foci, ${ }^{6-8}$ so it is still necessary to investigate the surgical benefits in patients with LACC.

About $80 \%$ of new cervical carcinoma cases are diagnosed in developing countries, ${ }^{9}$ and although $72 \%$ of patients with FIGO stage IIB cervical carcinoma are treated with definitive radiotherapy according to the annual FIGO report, in most high-risk areas of Asia, the majority of these patients undergo radical hysterectomy. For example, $62.7 \%$ of such patients have been reported to receive surgery-based therapy in Japan. ${ }^{10-12}$ It is becoming increasingly important to compare the benefit of surgery in patients with FIGO stage IIB cervical carcinoma, especially in high-risk Southeast Asia, with the disease reduction achieved in European countries and in the US.

In this paper, we report the results of a comparison between concurrent chemoradiotherapy followed by radical surgery and concurrent chemoradiotherapy followed by high-dose-rate intracavitary brachytherapy in 240 patients with FIGO stage IIB cervical carcinoma in high-risk regions of the People's Republic of China. Long-term survival and late toxicity were observed, and some prognostic factors are identified.

\section{Materials and methods Selection criteria}

From November 2004 to November 2011, 240 patients with FIGO stage IIB cervical carcinoma were treated at the Department of Radiation Oncology, Xijing Hospital, Fourth
Military Medical University, People's Republic of China. All patients had a Karnofsky performance status $\geq 70$ and had no history of other malignancy or treatment for carcinoma. Pretreatment evaluations comprised the patient's medical history, a blood count, liver and renal function tests, a gynecologic examination, cervical biopsy, chest X-ray, transvaginal ultrasound, abdominal ultrasonography, and pelvic magnetic resonance imaging (MRI). Tumor sizes were basically determined by MRI imaging. Patients had to have adequate bone marrow, hepatic, and renal function with the following criteria: a leukocyte count $\geq 3,000 / \mathrm{mm}^{3}$, a platelet count $\geq 100,000 / \mathrm{mm}^{3}$, hemoglobin $\geq 10 \mathrm{~g} / \mathrm{dL}$, total bilirubin $\leq 1.5 \mathrm{mg} / \mathrm{dL}$, aspartate aminotransferase/alanine aminotransferase no more than twice the upper limit of normal, and serum creatinine $\leq 1.5 \mathrm{mg} / \mathrm{dL}$.

\section{Treatment modalities}

\section{Concurrent chemoradiotherapy}

Pelvic external beam radiotherapy (EBRT) was delivered using three-dimensional conformal radiation techniques and $6 \mathrm{mV}$ or $15 \mathrm{mV}$ photons using a linear accelerator, ie, a Varian 23EX (Varian Medical Systems, Palo Alto, CA, USA) or a 600 C/D (Varian Medical Systems). Patients were immobilized on a custom vacuum mattress in the supine position and underwent a computed tomography simulation scan using a PQS and an AcQSim (Philips Health Care, Andover, MA, USA) with intravenous contrast and a $5 \mathrm{~mm}$ slice thickness. The clinical target volume included the gross tumor, cervix, uterus, parametrium, upper part of the vagina to $3 \mathrm{~cm}$ below the tumor invasion (according to the T2 MRI image), and regional lymph nodes (common, external, internal iliac, obturator, and presacral). Positive lymph nodes were delineated as having a minimum diameter $\geq 1 \mathrm{~cm}$, being necrotic, or involving multiple lymph nodes. The planning target volume was defined by uniform three-dimensional expansion around the clinical target volume, using $7 \mathrm{~mm}$ margins around the lymph nodes, $10 \mathrm{~mm}$ around the vagina and parametrium, and $15 \mathrm{~mm}$ around the cervix and gross disease. Treatment was designed and computed using the PLATO system version 2.7.5. The EBRT dose was 40-50 Gy in 20-25 fractions. Radiotherapy was withheld when hematologic toxicity reached grade 4 or nonhematologic toxicity reached grade 3-4. Radiotherapy was resumed when hematologic and nonhematologic toxicity recovered to grade 2 .

Concurrent chemotherapy was administered in 4-6 cycles by an intravenous infusion of weekly cisplatin $\left(40 \mathrm{mg} / \mathrm{m}^{2}\right)$ during pelvic EBRT. Chemotherapy was withheld under the following conditions: white blood cell count $<2.0 \times 10^{9} / \mathrm{L}$, 
absolute neutrophil count $<1.0 \times 10^{9} / \mathrm{L}$, platelet count $<50 \times 10^{9} / \mathrm{L}$, or grade $3-4$ radiation enteritis or cystitis.

All 240 patients had a good response to standard treatment, with a decrease in tumor volume of at least $50 \%$ according to MRI on completion of concurrent chemoradiotherapy. The patients were divided into two groups, ie, for radical surgery or for high-dose-rate intracavitary brachytherapy. The choice of therapeutic modality depended mainly on patient preference, except in those who could not undergo surgery because of severe cardiac or pulmonary disease. Three such patients received intracavitary brachytherapy because of comorbidity, comprising two with a history of arrhythmia and one older patient (aged 71 years) with a permanent cardiac pacemaker.

\section{Concurrent chemoradiotherapy followed by radical surgery (group I)}

Group 1 patients $(n=119)$ underwent radical abdominal hysterectomy and pelvic lymphadenectomy. The interval between preoperative concurrent chemoradiotherapy and radical surgery was 2-3 weeks. The resected primary and lymph node specimens were processed into sections for hematoxylin and eosin staining and pathologic observation. The pathologic response to neoadjuvant therapy was evaluated based on histopathologic examination of the resected specimens, ie, uterus, vaginal cuff, parametrium, and pelvic lymph nodes.

\section{Radical radiotherapy associated with concurrent chemotherapy (group 2)}

Group 2 patients $(n=121)$ underwent radical radiotherapy. The whole pelvic EBRT dose was boosted to 50 Gy in 25 fractions, and positive lymph nodes were boosted to 64-66 Gy. Computed tomography-based image-guided brachytherapy was applied at the conclusion of pelvic EBRT, and concurrently with boost radiation to the lymph nodes. An Ir-192 source was used for high-dose-rate intracavitary brachytherapy. high-dose-rate intracavitary brachytherapy was delivered with $28-35$ Gy in 4-5 fractions to $90 \%$ of the high-risk clinical target volume $\left(\mathrm{D}_{90}\right)$, using interstitial implantation or an intracavity applicator (microSelectronHDR Ir-192 set or Fletcher applicator set, Nucletron, Veenendaal, The Netherlands). The treatment procedure for patients in group 1 and group 2 is shown schematically in Figure 1.

\section{Follow-up}

Patients were followed up at 3-monthly intervals in the first year and at 6-monthly intervals thereafter with regular gynecologic examination, laboratory studies (blood count, liver and renal function tests), transvaginal ultrasound, abdominal ultrasonography, superficial lymph node examination, and radiographic studies, including chest computed tomography and/or pelvic MRI.

Toxicity assessment was performed according to the RTOG/European Organization for Research and Treatment for Carcinoma late radiation morbidity scoring scheme. ${ }^{13}$ Surgical complications were classified using the Chassagne grading system. ${ }^{14,15}$ Overall survival and progression-free survival rates were calculated from the date of surgery or completion of intracavitary brachytherapy to the last follow-up date.

\section{Statistical analysis}

Statistical analysis was performed using Statistical Package for the Social Sciences version 17 software (SPSS Inc,

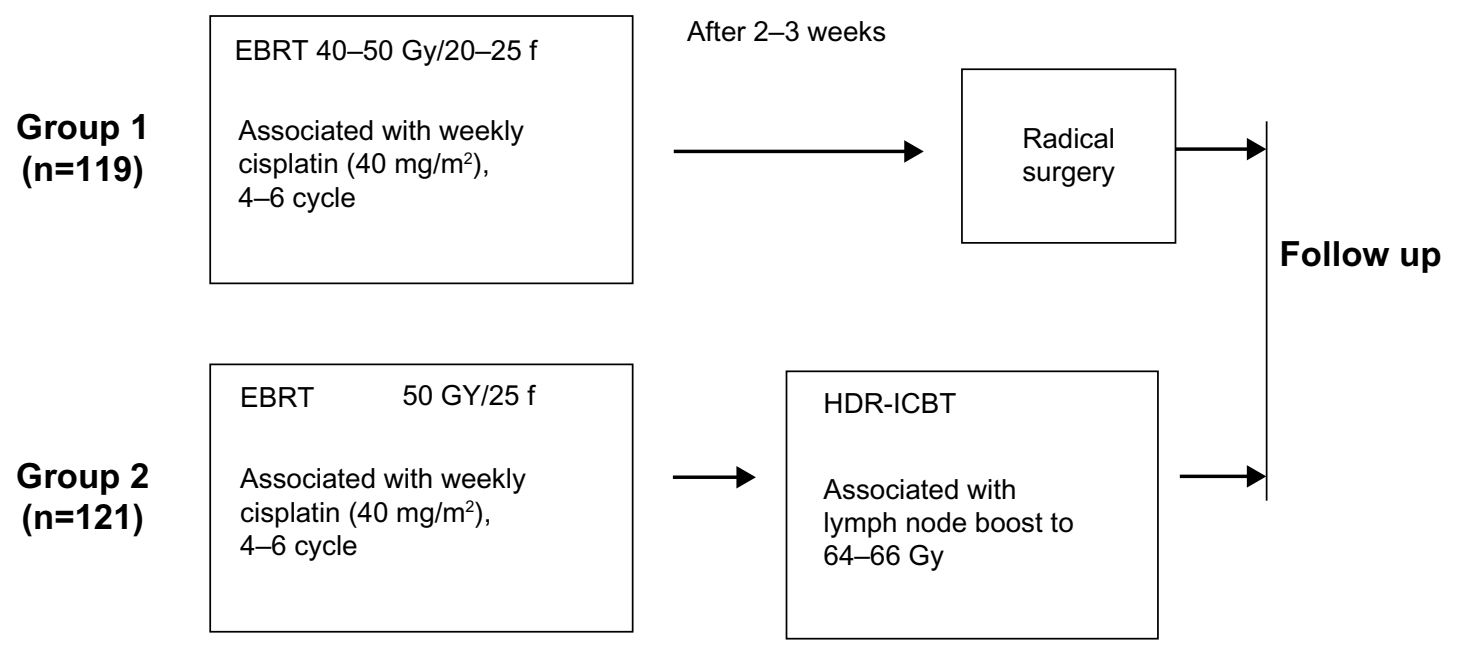

Figure I Schematic diagram showing treatment procedure for 240 patients in group I and group 2.

Abbreviations: EBRT, external beam radiotherapy; HDR-ICBT, high-dose-rate intracavitary brachytherapy. 
Chicago, IL, USA). Overall survival and progression-free survival rates were computed by the Kaplan-Meier method and are shown. Differences in the survival curves were estimated using the log-rank test. Multivariate analysis was performed by linear logistic regression. Other estimations were performed using the chi-square test. $P$-values less than 0.05 were considered to be statistically significant.

\section{Results}

\section{Patient characteristics}

The median follow-up is 36 (range 2-75) months. There are 118 patients with a follow-up period of more than 3 years (61 patients in group 1 and 57 patients in group 2). The median age is 45 (range 25-71) years. One hundred and nineteen patients received concurrent chemoradiotherapy followed by radical surgery in group 1, and 121 patients received radical radiotherapy with concurrent chemotherapy (weekly cisplatin $40 \mathrm{mg} / \mathrm{m}^{2}$ ) in group 2 . Of note, none of the patients in group 1 accepted postoperative adjuvant therapy. An overall treatment time of more than 56 days has been correlated with a bad prognosis. All patients in group 1 completed their full treatment in 56 days. However, in group 2, overall treatment time ranged from 45 to 99 days, with a median of 60 days.
No patient had an extension of overall treatment time because of severe toxicity. The main reason for extension of overall treatment time in group 2 was socioeconomic problems. Patient characteristics are shown in Table 1.

\section{Survival outcomes}

Concurrent chemoradiotherapy followed by radical surgery in group 1 showed a survival benefit compared with group 2 (3-year overall survival 94.9\% versus $84.6 \%, P=0.011$; 3 -year progression-free survival $91.0 \%$ versus $81.8 \%, P=0.049$ ) (Figure 2). Three-year local pelvic control was $94.6 \%$ in group 1 and $93.3 \%$ in group 2 , respectively $(P=0.325)$.

In group 1 , local regional failure developed in five patients $(4.20 \%)$. Distant metastasis developed in seven patients $(5.88 \%)$. One patient developed pelvic recurrence and liver metastasis concurrently. One patient in group 1 developed postoperative intestinal obstruction. In group 2, local regional failure developed in eight patients $(6.61 \%)$ and distant metastasis developed in 13 patients $(10.74 \%)$. Of note, one patient died from a second primary tumor (leukemia) in group 2. Distant metastasis was still the main reason for treatment failure in these two groups. No patients died from non-tumor-related diseases or reasons.

Table I Patient characteristics

\begin{tabular}{|c|c|c|c|c|}
\hline \multirow[t]{2}{*}{ Characteristics } & \multirow{2}{*}{$\frac{\text { Group I (n=I I 9) }}{\text { Patients, n (\%) }}$} & \multirow{2}{*}{$\frac{\text { Group } 2(n=121)}{\text { Patients, } n(\%)}$} & \multirow[t]{2}{*}{$\chi^{2}$} & \multirow[t]{2}{*}{$P$-value } \\
\hline & & & & \\
\hline Median age (range, years) & $45,27-65$ & $52,25-7 \mid$ & & \\
\hline$>35$ & 110 (92.44\%) & 117 (96.69\%) & 2.122 & 0.165 \\
\hline$\leq 35$ & $9(7.56 \%)$ & $4(3.31 \%)$ & & \\
\hline \multicolumn{5}{|l|}{ KPS score } \\
\hline 90 & $46(38.66 \%)$ & 42 (34.7।\%) & 0.402 & 0.592 \\
\hline 80 & $66(55.46 \%)$ & $73(60.33 \%)$ & 0.583 & 0.513 \\
\hline 70 & $7(5.88 \%)$ & $6(4.96 \%)$ & 0.100 & 0.783 \\
\hline Tumor diameter $(\mathrm{cm})$ & I.2-8.0, $4.5,4.43$ & $1.5-6.5,4.0,3.88$ & & \\
\hline \multicolumn{5}{|l|}{ (range, median, mean) } \\
\hline$\geq 4 \mathrm{~cm}$ & 93 (78.15\%) & $74(61.16 \%)$ & 8.187 & $0.005^{* *}$ \\
\hline$<4 \mathrm{~cm}$ & $26(21.85 \%)$ & 47 (38.84\%) & & \\
\hline$\geq 6 \mathrm{~cm}$ & II (9.24\%) & $7(5.79 \%)$ & 1.034 & 0.337 \\
\hline$<6 \mathrm{~cm}$ & 108 (90.76\%) & II 4 (94.21\%) & & \\
\hline \multicolumn{5}{|l|}{ Pathologic types } \\
\hline SCC & 112 (94.12\%) & II 6 (95.87\%) & 0.387 & 0.569 \\
\hline non-SCC & $7(5.88 \%)$ & $5(4.13 \%)$ & & \\
\hline \multicolumn{5}{|l|}{ LN involvement } \\
\hline+ & $13(10.92 \%)$ & 24 (19.83\%) & 3.653 & 0.073 \\
\hline- & $106(89.08 \%)$ & $97(80.17 \%)$ & & \\
\hline \multicolumn{5}{|l|}{ EBRT dose } \\
\hline 40-45 Gy & 39 & 28 & 2.766 & 0.114 \\
\hline 46-50 Gy & 80 & 93 & & \\
\hline $\begin{array}{l}\text { Median follow-up } \\
\text { (range, months) }\end{array}$ & $36,7-75$ & $30,2-70$ & & 0.173 \\
\hline
\end{tabular}

Abbreviations: EBRT, external beam radiotherapy; KPS, Karnofsky performance status; SCC, squamous cell carcinoma; LN, lymph node. ** $P<0.01$. 
Table 2 Treatment failure patterns in group 1 and group 2 patients

\begin{tabular}{|c|c|c|c|c|}
\hline & $\begin{array}{l}\text { Group I } \\
(n=I \mid 9)\end{array}$ & $\begin{array}{l}\text { Group } 2 \\
(n=121)\end{array}$ & $\chi^{2}$ & $P$-value \\
\hline & $\begin{array}{l}\text { Patients, } \\
\text { n (\%) }\end{array}$ & $\begin{array}{l}\text { Patients, } \\
\text { n (\%) }\end{array}$ & & \\
\hline Pelvic recurrence & $5(4.20 \%)$ & $8(6.61 \%)$ & 0.680 & 0.570 \\
\hline $\begin{array}{l}\text { Total distant } \\
\text { metastasis }\end{array}$ & 7 (5.88\%) & 13 (I0.74\%) & 1.856 & 0.243 \\
\hline Lung metastasis & $3(2.52 \%)$ & $6(4.96 \%)$ & & \\
\hline Bone metastasis & I (0.84\%) & $4(3.31 \%)$ & & \\
\hline $\begin{array}{l}\text { Lymph node } \\
\text { metastasis }\end{array}$ & $2(1.68 \%)$ & $4(3.31 \%)$ & & \\
\hline Liver metastasis & I (0.84\%) & 0 & & \\
\hline $\begin{array}{l}\text { Second primary } \\
\text { tumor }\end{array}$ & 0 & I (0.83\%) & 0.988 & 1.000 \\
\hline $\begin{array}{l}\text { Total treatment } \\
\text { failure }\end{array}$ & $1 \mathrm{I} *(9.24 \%)$ & $22^{\#}(18.18 \%)$ & 4.042 & 0.060 \\
\hline
\end{tabular}

Treatment failure patterns in the two groups are summarized in Table 2.

\section{Tumor size as a key prognostic factor}

Prognostic factors in group 1 were: age $(\leq 35$ years versus $>35$ years); 3 -year progression-free survival ( $71.4 \%$ versus $90.9 \%, P=0.037$ ); tumor diameter ( $\geq 6 \mathrm{~cm}$ versus $<6 \mathrm{~cm})$; and 3-year progression-free survival $(60.6 \%$ versus $92.9 \%$, $P=0.004)$. Prognostic factors in group 2 were: tumor diameter ( $\geq 4 \mathrm{~cm}$ versus $<4 \mathrm{~cm}$ ); 3 -year overall survival (78.0\% versus $94.8 \%, P=0.043$ ); tumor diameter ( $\geq 6 \mathrm{~cm}$ versus $<6 \mathrm{~cm}$ ); 3-year progression-free survival $(42.9 \%$ versus $84.2 \%$, $P=0.032)$; and 3 -year overall survival $(42.9 \%$ versus $87.1 \%$, $P=0.013)$. These data are shown in Tables 3, 4 and Figure 3 .

\section{Postoperative pathologic response in group 1}

Pathologic responses to neoadjuvant therapy were further evaluated in group 1 based on histopathologic examination. The postoperative pathologic response included complete response, partial response, and residual carcinoma. Complete response was defined as complete disappearance of all macroscopic and microscopic disease; partial response as presence of persistent atypical cells or cervical intraepithelial neoplasia; and residual carcinoma as macroscopic and/or microscopic residual disease. The complete response rate was $27.73 \%$ (33/119), the partial response rate was $41.18 \%$ (49/119), and the residual carcinoma rate was $31.09 \%$ $(37 / 119)$. The total effective pathologic alleviation rate was $68.91 \%$, including complete responses $(33 / 119,27.73 \%)$ and partial responses $(49 / 119,41.18 \%)$.

\section{Late toxicity}

Fifty patients $(50 / 119,42.02 \%)$ in group 1 and 46 patients $(46 / 121,38.02 \%)$ in group 2 suffered late complications (Table 5). Of note, 12 patients in group 1 suffered from at least two types of complications concurrently, and eight patients in group 2 suffered from at least two types of complications concurrently. There was no statistically significant difference in total late complication rate between the two

Table 3 Correlations between clinical/pathologic factors and survival outcome in group I

\begin{tabular}{|c|c|c|c|c|c|c|c|c|c|c|c|}
\hline \multirow{2}{*}{$\begin{array}{l}\text { Prognostic } \\
\text { factors }\end{array}$} & \multirow{2}{*}{$\begin{array}{l}\text { Patients, } \\
\text { n }\end{array}$} & \multirow{2}{*}{$\begin{array}{l}\text { 3-year } \\
\text { OS }\end{array}$} & \multirow[t]{2}{*}{$P$-value } & \multirow{2}{*}{$\begin{array}{l}\operatorname{Exp}(B) \\
\text { HR }\end{array}$} & \multicolumn{2}{|l|}{$95 \% \mathrm{Cl}$} & \multirow{2}{*}{$\begin{array}{l}\text { 3-year } \\
\text { PFS }\end{array}$} & \multirow[t]{2}{*}{$P$-value } & \multirow{2}{*}{$\begin{array}{l}\operatorname{Exp}(B) \\
\text { HR }\end{array}$} & \multicolumn{2}{|l|}{$95 \% \mathrm{Cl}$} \\
\hline & & & & & Lower & Upper & & & & Lower & Upper \\
\hline \multicolumn{12}{|l|}{ Age } \\
\hline$\leq 35$ years & 9 & $88.9 \%$ & 0.258 & 0.278 & 0.030 & 2.552 & $74.1 \%$ & $0.037^{*}$ & 0.167 & 0.031 & 0.894 \\
\hline$>35$ years & 110 & $95.4 \%$ & & & & & $90.9 \%$ & & & & \\
\hline \multicolumn{12}{|c|}{ Primary tumor } \\
\hline$<4 \mathrm{~cm}$ & 26 & $96.2 \%$ & 0.797 & 1.353 & 0.135 & 13.562 & $96.0 \%$ & 0.653 & 1.468 & 0.275 & 7.844 \\
\hline$\geq 4 \mathrm{~cm}$ & 93 & $94.6 \%$ & & & & & $87.8 \%$ & & & & \\
\hline \multicolumn{12}{|c|}{ Primary tumor } \\
\hline$<6 \mathrm{~cm}$ & 108 & $96.3 \%$ & 0.122 & 0.232 & 0.038 & 1.405 & $92.9 \%$ & $0.004^{* *}$ & 0.140 & 0.036 & 0.542 \\
\hline$\geq 6 \mathrm{~cm}$ & II & $81.8 \%$ & & & & & $60.6 \%$ & & & & \\
\hline \multicolumn{12}{|l|}{ EBRT dose } \\
\hline $40-45$ Gy & 39 & $87.5 \%$ & 0.961 & 0.000 & 0.000 & 3.030 & $87.1 \%$ & 0.432 & 0.415 & 0.046 & 3.725 \\
\hline 46-50 Gy & 80 & $96.6 \%$ & & & & & $93.3 \%$ & & & & \\
\hline \multicolumn{12}{|c|}{ LN involvement } \\
\hline+ & 13 & $92.3 \%$ & 0.145 & 0.296 & 0.057 & 1.524 & $80.8 \%$ & 0.422 & 0.536 & 0.117 & 2.455 \\
\hline- & 106 & $95.2 \%$ & & & & & $90.4 \%$ & & & & \\
\hline
\end{tabular}

Abbreviations: $\mathrm{Cl}$, confidence interval; HR, hazard ratio; SCC, squamous cell carcinoma; EBRT, external beam radiotherapy; LN, lymph node; OS, overall survival; PFS, progression-free survival $* P<0.05 ; * * P<0.01$. 
Table 4 Correlations between clinical/pathologic factors and survival outcome in group 2

\begin{tabular}{|c|c|c|c|c|c|c|c|c|c|c|c|}
\hline \multirow{2}{*}{$\begin{array}{l}\text { Prognostic } \\
\text { factors }\end{array}$} & \multirow[t]{2}{*}{ Patients, $n$} & \multirow{2}{*}{$\begin{array}{l}\text { 3-year } \\
\text { OS }\end{array}$} & \multirow[t]{2}{*}{$P$-value } & \multirow{2}{*}{$\begin{array}{l}\operatorname{Exp}(B) \\
\text { HR }\end{array}$} & \multicolumn{2}{|l|}{$95 \% \mathrm{Cl}$} & \multirow{2}{*}{$\begin{array}{l}\text { 3-year } \\
\text { PFS }\end{array}$} & \multirow[t]{2}{*}{$P$-value } & \multirow{2}{*}{$\begin{array}{l}\operatorname{Exp}(B) \\
\text { HR }\end{array}$} & \multicolumn{2}{|l|}{$95 \% \mathrm{Cl}$} \\
\hline & & & & & Lower & Upper & & & & Lower & Upper \\
\hline \multicolumn{12}{|l|}{ Age } \\
\hline$\leq 35$ years & 4 & $75.0 \%$ & 0.590 & 1.849 & 0.198 & 17.265 & $75.0 \%$ & 0.619 & 1.745 & 0.195 & 15.648 \\
\hline$>35$ years & 117 & $84.9 \%$ & & & & & $82.0 \%$ & & & & \\
\hline \multicolumn{12}{|c|}{ Primary tumor } \\
\hline$<4 \mathrm{~cm}$ & 47 & $94.8 \%$ & $0.043^{*}$ & 0.211 & 0.047 & 0.949 & $89.4 \%$ & 0.127 & 0.444 & 0.157 & 1.258 \\
\hline$\geq 4 \mathrm{~cm}$ & 74 & $78.0 \%$ & & & & & $77.0 \%$ & & & & \\
\hline \multicolumn{12}{|c|}{ Primary tumor } \\
\hline$<6 \mathrm{~cm}$ & 114 & $87.1 \%$ & $0.013^{*}$ & 0.179 & 0.046 & 0.700 & $84.2 \%$ & $0.032 *$ & 0.226 & 0.058 & 0.882 \\
\hline$\geq 6 \mathrm{~cm}$ & 7 & $42.9 \%$ & & & & & $42.9 \%$ & & & & \\
\hline \multicolumn{12}{|l|}{ OTT } \\
\hline$\geq 56$ days & 44 & $83.1 \%$ & 0.347 & 0.602 & $0.20 \mathrm{I}$ & 1.732 & $79.2 \%$ & 0.202 & 0.523 & 0.193 & 1.416 \\
\hline$<56$ days & 77 & $86.4 \%$ & & & & & $86.2 \%$ & & & & \\
\hline \multicolumn{12}{|c|}{ LN involvement } \\
\hline+ & 24 & $86.4 \%$ & 0.700 & 1.276 & 0.369 & 4.409 & $80.3 \%$ & 0.431 & 1.632 & 0.483 & 5.515 \\
\hline- & 97 & $87.5 \%$ & & & & & $84.2 \%$ & & & & \\
\hline
\end{tabular}

Abbreviations: $\mathrm{Cl}$, confidence interval; HR, hazard ratio; SCC, squamous cell carcinoma; OTT, overall treatment time; LN, lymph node; OS, overall survival; OTT, overall treatment time; PFS, progression-free survival $* P<0.05$.

groups, but the composition showed obvious differences. The rate of leg edema in group 1 was higher than in group 2 (35.29\% versus $4.96 \%, P=0.000)$, and the radiation enteritis rate in group 2 was higher than in group 1 (30.58\% versus $5.04 \%, P=0.000)$. The data indicate that radiation enteritis was more common in group 2 and might be correlated with intracavitary brachytherapy and boost radiation to the lymph nodes. The median number of lymph node dissections was
18 (range 6-42) in group 1, and after subgroup analysis, the data showed that leg edema occurred more frequently when pelvic lymph node dissection was $\geq 20$ (55.56\% versus $29.79 \%, P=0.022)$.

\section{Discussion}

The treatment strategy used for LACC has changed over the past two decades. Combined treatment modalities have
A

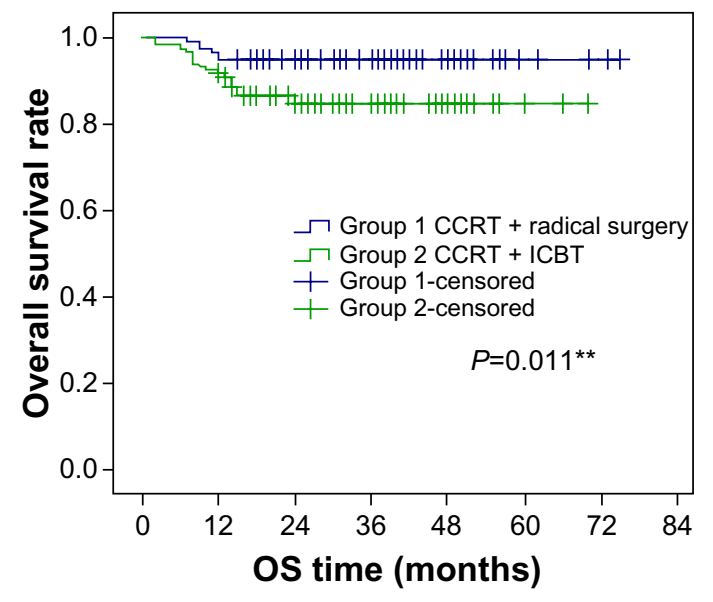

$\begin{array}{rrrrrr} & 12 \mathrm{~m} & 24 \mathrm{~m} & 36 \mathrm{~m} & 48 \mathrm{~m} & 60 \mathrm{~m} \\ \text { Group 1 } & 94.9 \% & 94.9 \% & 94.9 \% & 94.9 \% & 94.9 \% \\ \text { Group 2 } & 91.7 \% & 84.6 \% & 84.6 \% & 84.6 \% & 84.6 \%\end{array}$
B

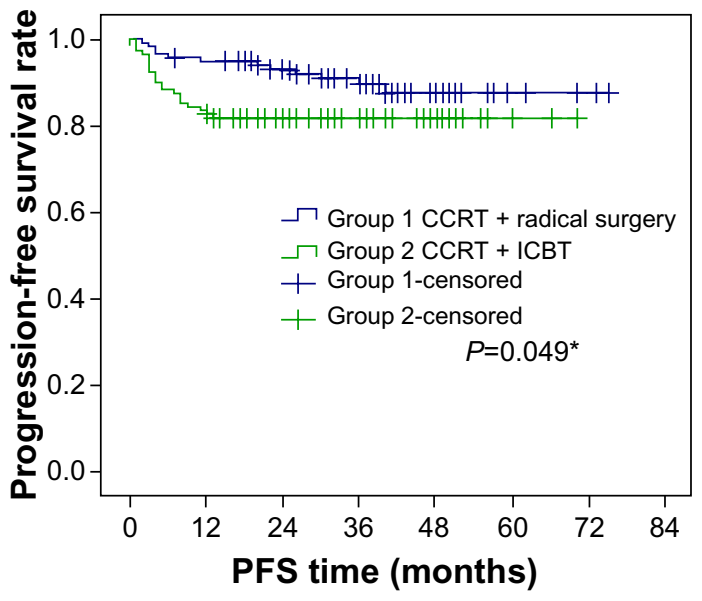

$\begin{array}{rrrrr}12 \mathrm{~m} & 24 \mathrm{~m} & 36 \mathrm{~m} & 48 \mathrm{~m} & 60 \mathrm{~m} \\ \text { Group 1 } 95.0 \% & 93.1 \% & 91.0 \% & 91.0 \% & 91.0 \% \\ \text { Group 2 } 90.1 \% & 81.8 \% & 81.8 \% & 81.8 \% & 81.8 \%\end{array}$

Figure 2 Overall survival and progression-free survival in group I and group 2 patients, respectively. Patients in group I show a survival benefit in comparison with those in group 2. Three-year overall survival in group I was $94.9 \%$ and in group 2 was $84.6 \%(* P=0.01 \mathrm{I})$. Three-year progression-free survival in group I was $91.0 \%$ and in group 2 was $81.8 \%(* P=0.049)$.

Abbreviations: OS, overall survival; PFS, progression-free survival; CCRT, concurrent chemoradiotherapy; ICBT, intracavitary brachytherapy. 
A

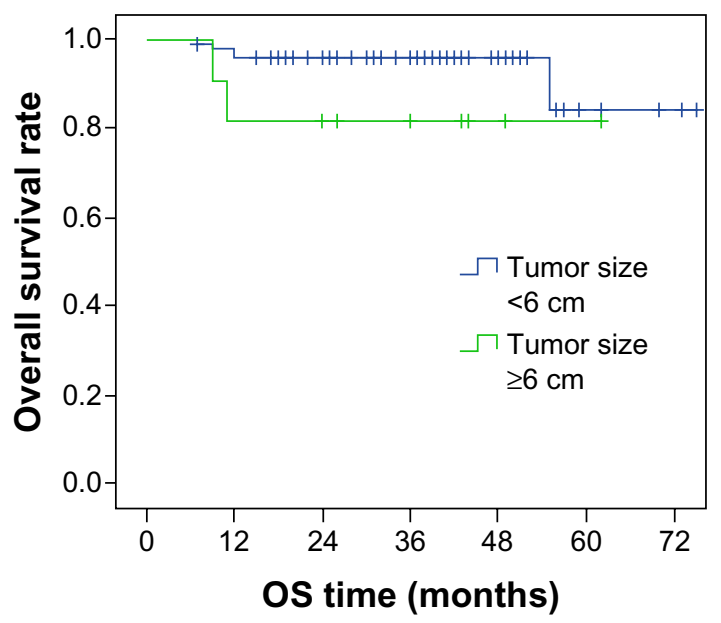

C

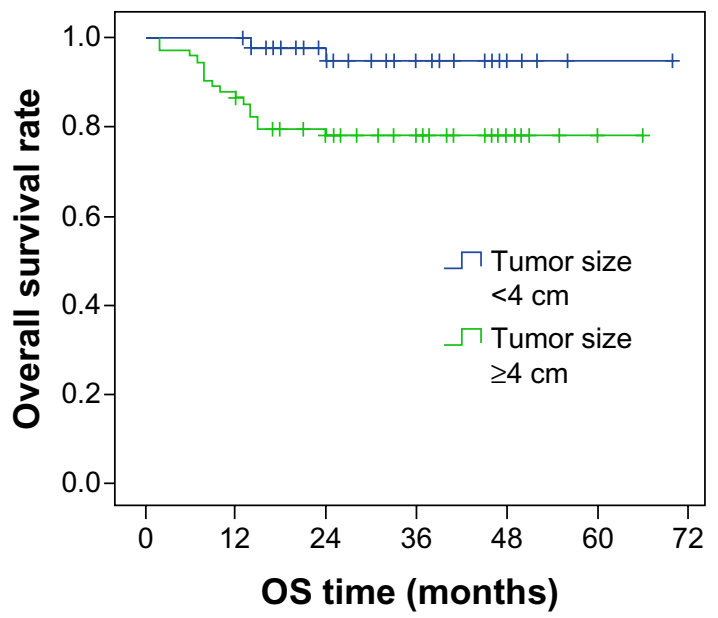

B

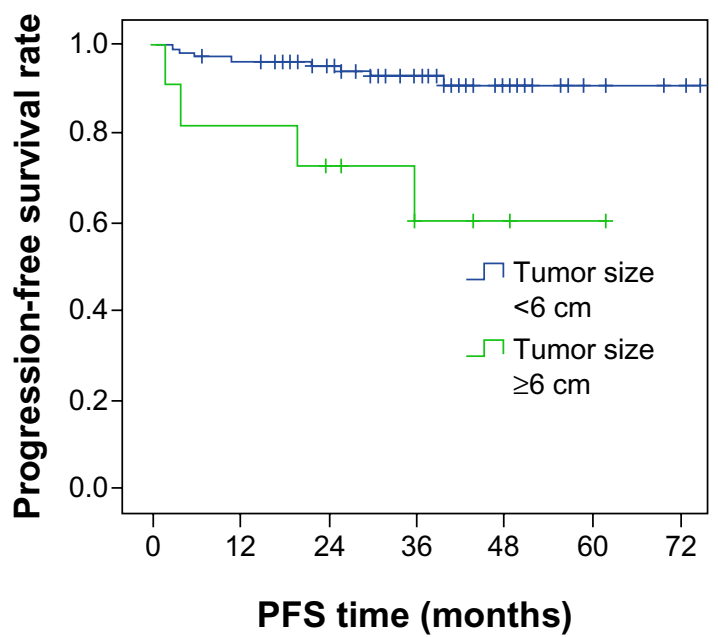

D

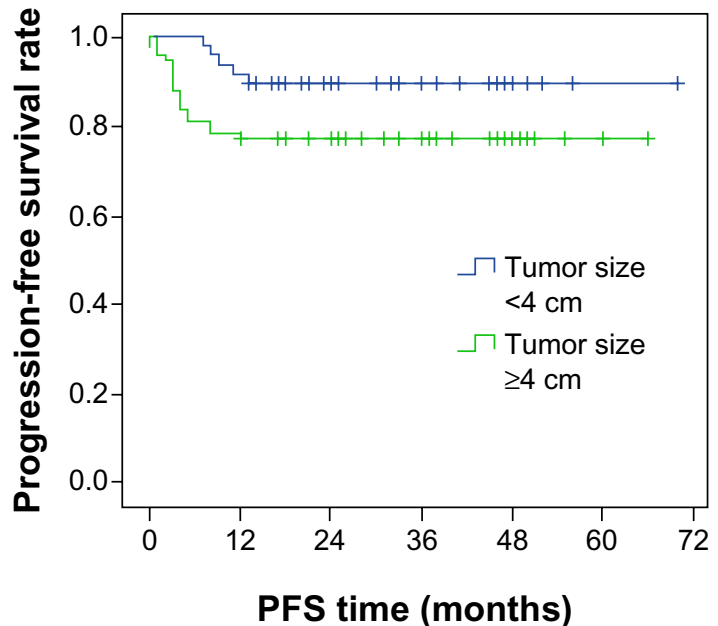

Figure 3 Overall survival and progression-free survival are shown regarding tumor size as a prognostic factor with a boundary of $6 \mathrm{~cm}$ in group I (A and B) (3-year overall survival, $P=0.122$; 3-year progression-free survival, $P=0.004$ ) and with a boundary of $4 \mathrm{~cm}$ in group 2 (C and $\mathbf{D})$ (3-year overall survival, $P=0.043 ; 3$-year progression-free survival, $P=0.127$ ).

Abbreviations: OS, overall survival; PFS, progression-free survival.

Table 5 Late complications in group I and group 2 patients

\begin{tabular}{|c|c|c|c|c|}
\hline $\begin{array}{l}\text { Late } \\
\text { complications }\end{array}$ & $\begin{array}{l}\begin{array}{l}\text { Group I } \\
(n=\mid \text { I9) }\end{array} \\
\text { Patients, } \\
n(\%)\end{array}$ & $\begin{array}{l}\begin{array}{l}\text { Group } 2 \\
(\mathrm{n}=12 \mathrm{I})\end{array} \\
\text { Patients, } \\
\mathrm{n}(\%)\end{array}$ & $\chi^{2}$ & $P$-value \\
\hline Irradiation enteritis & $6(5.04 \%)$ & 37 (30.58\%) & 26.603 & $0.000^{* *}$ \\
\hline Grade I & 6 & 23 & & \\
\hline Grade 2 & 0 & 14 & & \\
\hline Irradiation cystitis & $10(8.40 \%)$ & $10(8.26 \%)$ & 0.002 & 1.000 \\
\hline Grade I & 10 & 7 & & \\
\hline Grade 2 & - & 3 & & \\
\hline Leg edema & 42 (35.29\%) & $6(4.96 \%)$ & 34.507 & $0.000 * *$ \\
\hline Grade I & 32 & 4 & & \\
\hline Grade 2 & 10 & 2 & & \\
\hline Uronephrosis & $5(4.20 \%)$ & I (0.83\%) & 2.804 & 0.118 \\
\hline Thrombus & $3(2.52 \%)$ & 0 & 3.089 & 0.120 \\
\hline $\begin{array}{l}\text { Intestinal } \\
\text { obstruction }\end{array}$ & I (0.84\%) & 0 & 1.021 & 0.496 \\
\hline
\end{tabular}

Note: $* * P<0.0$ I. focused on radical radiotherapy associated with concurrent chemotherapy, preoperative concurrent chemoradiotherapy with completion surgery, and neoadjuvant chemotherapy followed by completion surgery in recent years. ${ }^{16-20}$ According to the FIGO annual report of $2003,72 \%$ of patients with FIGO stage IIB cervical carcinoma were treated with definitive radiotherapy. ${ }^{12}$ In contrast, surgery is preferentially utilized over radiotherapy in most high-risk Southeast Asian areas, so it is still important to explore the surgical benefit for LACC patients. In this retrospective study, we used patients who underwent radical radiotherapy associated with concurrent chemotherapy as the historical concurrent control group. Concurrent chemoradiotherapy followed by radical surgery showed a survival benefit in patients with FIGO stage IIB cervical carcinoma. 
Both radical radiotherapy and surgery can destroy malignant cervical and paracervical cells as well as regional lymph nodes. However, surgery is recommended in several reports because it can remove chemoradiotherapy-resistant foci. ${ }^{7,21,22}$ For example, Houvenaeghel et $\mathrm{al}^{22}$ reported on 30 patients with advanced cervical carcinoma and bulky residual disease $(\geq 2 \mathrm{~cm}$ ) after concurrent chemoradiotherapy who accepted curative surgery. In the absence of para-aortic involvement, $80 \%$ of these patients were still alive 5 years after curative surgery. This result suggests that adjuvant surgery might improve the outcome of patients with bulky residual disease after concurrent chemoradiotherapy. Lèguevaque et $\mathrm{al}^{18}$ reported that additional surgery after concurrent chemoradiotherapy followed by brachytherapy improved disease-free survival by reducing pelvic recurrence. In their study, disease-free survival and overall survival were compared in 111 patients with FIGO stage IB1 to IVA disease (mainly FIGO stage IIB [55/111, 49.5\%]). The recurrence rate was lower in patients who underwent completion surgery with less than $50 \%$ residual tumor than in patients with a good response to concurrent chemoradiotherapy who were therefore treated conservatively $(22.4 \%$ versus $36.4 \%, P=0.01)$. This research suggests that surgery could have a survival benefit for patients who do not have a good response to concurrent chemoradiotherapy by removing chemoradiotherapy-resistant foci.

Pelvic recurrence and distant metastasis could be decreased as a consequence of removal of chemoradiotherapy-resistant foci. Carcopino et $\mathrm{al}^{23}$ reported on the survival outcome in patients with FIGO stage IB-IVA cervical carcinoma who underwent surgery after concurrent chemoradiotherapy. Surgery was curative in 127 cases and pelvic control was achieved in 114 cases. Pelvic control and survival rates were equivalent in patients with stage IB-II and III-IVA disease, suggesting that adjuvant surgery following chemoradiotherapy may improve local control, enabling patients with stage III-IVA disease to have a survival outcome comparable with that of patients with stage IB-II disease. In our study, surgical-based treatment (group 1) also achieved better pelvic control and an improved survival outcome, and this result is consistent with the other published research. For example, Kato et al $^{24}$ reported a multi-institutional Phase II clinical study from East and Southeast Asia in which 120 patients with LACC (60 with bulky stage IIB disease) accepted concurrent chemoradiotherapy using five cycles of weekly cisplatin. Radiotherapy consisted of pelvic EBRT (total dose 50 Gy) and intracavitary brachytherapy. This treatment modality was similar to that used for group 2 in our study. In that Phase II clinical study, the 2-year pelvic tumor control rate for patients with bulky stage IIB disease was $94.2 \%$. In our study, the 2 -year pelvic tumor control rate in group $2(93.3 \%)$ was comparable with that in the report by Kato et al $(94.2 \%)$.

Distant metastasis is still the main reason for treatment failure. Recent research suggests that local pelvic failure is associated with a higher risk of metastasis. In our study, distant metastasis occurred more frequently in group $2(13 / 121$, $10.74 \%)$ than in group $1(7 / 119,5.88 \%)$. We therefore deduced that radical surgery could decrease the risk of metastasis by clearing the pelvic lymph nodes, and could also bring about a survival benefit by removal of radiochemotherapyresistant foci, thereby improving pelvic control in patients with FIGO stage IIB cervical carcinoma.

Size of the primary tumor is an independent risk factor for local regional failure and survival according to multivariate analysis. ${ }^{25}$ In a previous study, we showed that preoperative concurrent chemoradiotherapy can improve progression-free survival and overall survival compared with radiotherapy alone when the tumor diameter is less than $5 \mathrm{~cm}$, indicating that the beneficial effect of concurrent chemotherapy might be limited or depend on tumor size. ${ }^{26}$ In the present work, the size of the primary tumor was still an independent risk factor in group 1 patients with a boundary of $6 \mathrm{~cm}$ and those in group 2 with a boundary of $4 \mathrm{~cm}$. The mean tumor diameter in group $1(4.43 \mathrm{~cm})$ was larger than that in group $2(3.88 \mathrm{~cm})$, and the difference in tumor size between these two groups was statistically significant for a boundary of $4 \mathrm{~cm}(P=0.005)$, but the survival outcome was better in group 1 . This result implies that surgical treatment could alleviate the adverse prognostic effect of primary tumor burden. Based on this difference, we deduced that radical surgery could bring about a survival benefit for patients with a bulky tumor burden by removing radiochemotherapy-resistant foci, which is consistent with other published research, ${ }^{18}$ but this conclusion needs to be validated in a large number of patients.

The use of a three-modality treatment can achieve survival outcomes compared with those achieved by exclusive use of chemoradiotherapy. However, one of the concerns about surgery following chemoradiotherapy is surgical morbidity. Tummers et $\mathrm{a}^{21}$ reported on the outcome of surgery after chemoradiotherapy (with intensity-modulated arc therapy) in 34 patients with LACC, and their complication rates are comparable with those of primary surgery for cervical carcinoma. In our study, the main complication in group 1 was leg edema, and the number of lymph node dissections 
$(\geq 20)$ is related to the risk of leg edema. Further study should focus on how to decrease the rate of lymph node metastasis, with a view to determining the feasibility and safety of individualized surgical treatment. We do believe that a new concept of radical surgery based on intraoperative findings might decrease the risk of surgical complications. ${ }^{27}$ The main complication in group 2 was radiation enteritis (30.58\% versus $5.04 \%, P=0.000$ ). The patient distribution between group 1 and group 2 with regard to EBRT dose was not significantly different (Table $1, P=0.114$ ), so it was deduced that the higher rate of radiation enteritis in group 2 might be related to intracavitary brachytherapy and boost radiation to the lymph nodes. Of 24 patients in group 2 who accepted boost radiation to the lymph nodes, seven (29.2\%) developed enteritis.

Overall treatment time is potentially a poor prognostic factor, with a boundary of 56 days for cervical carcinoma. All patients in group 1 received their full treatment in 56 days, with longer treatment courses occurring mainly in group 2 (median 60 days; range 45-99 days). According to research reported by Huang et al, tumor repopulation is indeed a problem. ${ }^{28}$ Accelerated tumor repopulation has significant implications in low-dose-rate brachytherapy, and dose escalation may be required to compensate for the effects of tumor repopulation if the course of radiation therapy is protracted. In our study, patients in group 2 accepted high-dose-rate but not low-dose-rate intracavitary brachytherapy after EBRT. On the other hand, EBRT dose compensation (0.6 Gy/day) was given to patients with a long EBRT treatment course.

The survival outcome did not show a statistically significant difference with regard to overall treatment time ( $\geq 56$ days versus $<56$ days) in group 2 . Considering the fact that the median follow-up has been relatively short (36 months), we shall continue our observations to validate this conclusion by extension of follow-up.

In conclusion, this study has shown that concurrent chemoradiotherapy followed by radical surgery could lead to a better survival outcome in comparison with that achieved by radical radiotherapy with weekly cisplatin in 240 patients with FIGO stage IIB cervical carcinoma. Late complications were acceptable and comparable between the two treatment groups. Tumor diameter might also represent a prognostic factor for these patients. In view of the weakness of any conclusion based on a single institution and retrospective experience, we plan to perform a randomized multicenter prospective study in a greater population of subjects to validate our conclusion.

\section{Acknowledgment}

This work was partially supported by grants from the National Science Foundation of China (81272346 and 30970862).

\section{Disclosure}

The authors report no conflicts of interest in this work.

\section{References}

1. Green JA, Kirwan JM, Tierney JF, et al. Survival and recurrence after concomitant chemotherapy and radiotherapy for carcinoma of the uterine cervix: a systematic review and meta-analysis. Lancet. 2001;358:781-786.

2. Lukka H, Hirte H, Fyles A, et al. Concurrent cisplatin-based chemotherapy plus radiotherapy for cervical carcinoma - meta analysis. Clin Oncol. 2002;14:203-212.

3. Jemal A, Murray T, Ward E, et al. Cancer statistics 2005. CA Cancer J Clin. 2005;55:10-30.

4. Ferrandina G, Legge F, Fagotti A, et al. Preoperative concomitant chemoradiotherapy in locally advanced cervical carcinoma: safety, outcome, and prognostic measures. Gynecol Oncol. 2007;107: S127-S132.

5. Mariagrazia D, Anna F, Gabriella F, et al. Preoperative chemoradiotherapy in locally advanced cervical carcinoma: long-term outcome and complications. Gynecol Oncol. 2005;99:S166-S170.

6. Houvenaeghel G, Lelievre L, Gonzague-Casabianca L, et al. Long-term survival after concomitant chemoradiotherapy prior to surgery in advanced cervical carcinoma. Gynecol Oncol. 2006;100: 338-343.

7. Macchia G, Ferrandina G, Deodato F, et al. Concomitant boost dose escalation plus large-field preoperative chemoradiotherapy in locally advanced carcinoma of the uterine cervix: results of a phase I study (LARA-CC-1). Gynecol Oncol. 2010;118:128-133.

8. Classe JM, Rauch P, Rodier JF, et al. Surgery after concurrent chemoradiotherapy and brachytherapy for the treatment of advanced cervical carcinoma: morbidity and outcome: results of a multicenter study of the GCCLCC (Group des Chirurgiens de Centre de Lutte contre le Carcinoma). Gynecol Oncol. 2006;102:523-529.

9. Mohar A, Frias-Mendivil M. Epidemiology of cervical carcinoma. Cancer Invest. 2000;18:584-590.

10. Benedet JL, Odicino F, Maisonneuve P, et al. Carcinoma of the cervix uteri. Int J Gynaecol Obstet. 2003;83 Suppl 1:41-78.

11. Undurraga M, Loubeyre P, Dubuisson JB, et al. Early-stage cervical carcinoma: is surgery better than radiotherapy? Expert Rev Anticancer Ther. 2010;10:451-460.

12. Mabuchi S, Okazawa M, Isohashi F, et al. Radical hysterectomy with adjuvant radiotherapy versus definitive radiotherapy alone for FIGO stage IIB cervical carcinoma. Gynecol Oncol. 2011;123:241-247.

13. Cox JD, Stetz J, Pajak TF. Toxicity criteria of the Radiation Therapy Oncology Group (RTOG) and the European Organization for Research and Treatment of Carcinoma (EORTC). Int J Radiat Oncol Biol Phys. 1995;31:1341-1346.

14. Chassagne D, Sismondi P, Horiot JC, et al. A glossary for reporting complications of treatment in gynecological carcinomas. Radiother Oncol. 1993;26:195-202.

15. Dindo D, Demartines N, Clavien PA. Classification of surgical complications: a new proposal with evaluation in a cohort of 6336 patients and results of a survey. Ann Surg. 2004;240:205-213.

16. Morice P, Rouanet P, Rey A, et al. Results of the GYNECO 02 study, an FNCLCC phase III trial comparing hysterectomy with no hysterectomy in patients with a (clinical and radiological) complete response after chemoradiotherapy therapy for stage IB2 or II cervical carcinoma. Oncologist. 2012;17:64-71. 
17. Motton S, Houvenaeghel G, Delannes M, et al. Results of surgery after concurrent chemoradiotherapy in advanced cervical carcinoma: comparison of extended hysterectomy and extrafascial hysterectomy. Int J Gynecol Cancer. 2010;20:268-275.

18. Lèguevaque $\mathrm{P}$, Motton S, Delannes $\mathrm{M}$, et al. Completion surgery or not after concurrent chemoradiotherapy for locally advanced cervical carcinoma? Eur J Obstet Gynecol Reprod Biol. 2011;155:188-192.

19. Shen Y, Yang L, Wang ZH. Treatment of early bulky cervical carcinoma with neoadjuvant paclitaxel, carboplatin and cisplatin prior to laparoscopic radical hysterectomy and pelvic lymphadenectomy. Oncol Lett. 2012;3:641-645.

20. Tummers P, Makar A, Vandecasteele K, et al. Completion surgery after intensity-modulated arc therapy in the treatment of locally advanced cervical carcinoma: feasibility, surgical outcome, and oncologic results. Int J Gynecol Cancer. 2013;23:877-883.

21. Tummers P, Makar A, Vandecasteele K, et al. Completion surgery after intensity-modulated arc therapy in the treatment of locally advanced cervical cancer: feasibility, surgical outcome, and oncologic results. Int J Gynecol Cancer. 2013 Jun;23(5):877-83.

22. Houvenaeghel GL, Lelievre M, Buttarelli, et al. Contribution of surgery in patients with bulky residual disease after chemoradiotherapy for advanced cervical carcinoma. Eur J Surg Oncol. 2007;33:498-503.

23. Carcopino X, Houvenaeghel G, Buttarelli M, et al. Equivalent survival in patients with advanced stage IB-II and III-IVA cervical carcinoma treated by adjuvant surgery following chemoradiotherapy. Eur J Surg Oncol. 2008;34:569-575.
24. Kato S, Ohno T, Thephamongkhol K, et al. Multi-institutional phase II clinical study of concurrent chemoradiotherapy for locally advanced cervical carcinoma in East and Southeast Asia. Int J Radiat Oncol Biol Phys. 2010;77:751-757.

25. Hirakawa M, Nagai Y, Toita T, et al. High-risk group for locoregional recurrence in patients with stage IB-IIB squamous cell carcinoma of the cervix treated with concurrent chemoradiotherapy. Anticancer Res. 2011;31:1437-1441.

26. Wei LC, Wang N, Shi M, et al. Clinical outcome observation of preoperative concurrent chemoradiotherapy/radiotherapy alone in 174 Chinese patients with local advanced cervical carcinoma. Onco Targets Ther. 2013;6:67-74.

27. Ferrandina G, Margariti PA, Smaniotto D, et al. Long-term analysis of clinical outcome and complications in locally advanced cervical cancer patients administered concomitant chemoradiation followed by radical surgery. Gynecol Oncol. 2010;119:404-410.

28. Huang Z, Mayry NA, Gao M, et al. Onset time of tumor repopulation for cervical cancer: first evidence from clinical data. Int J Radiat Oncol Bio Phys. 2012;84:478-484.
OncoTargets and Therapy

\section{Publish your work in this journal}

OncoTargets and Therapy is an international, peer-reviewed, open access journal focusing on the pathological basis of all cancers, potential targets for therapy and treatment protocols employed to improve the management of cancer patients. The journal also focuses on the impact of management programs and new therapeutic agents and protocols on

\section{Dovepress}

patient perspectives such as quality of life, adherence and satisfaction The manuscript management system is completely online and includes a very quick and fair peer-review system, which is all easy to use. Visit http://www.dovepress.com/testimonials.php to read real quotes from published authors. 